\title{
Exact dynamics of driven Brownian oscillators
}

\author{
Rui-Xue $\mathrm{Xu},{ }^{*}$ Bao-Ling Tian, and Jian $\mathrm{Xu}$ \\ Hefei National Laboratory for Physical Sciences at Microscale, \\ University of Science and Technology of China, Hefei, Anhui 230026, China \\ YiJing Yan \\ Department of Chemistry, Hong Kong University of Science and Technology, Kowloon, Hong Kong
}

(Dated: Submitted to J. Chem. Phys. on July 30, 2008)

\begin{abstract}
Exact quantum master equation for a driven Brownian oscillator system is constructed via a Wigner phase-space Gaussian wave packet approach. The interplay between external field and dissipation leads to this system an effective field correction that enhances the polarization. This cooperative property is resulted from an effective bath response to the external field applied on the system. It is important in the low-frequency driving and intermediate bath memory region. We demonstrate this non-Markovian effect on the linear response and nonlinear dynamics and analyze the results together with analytical asymptotic expressions.
\end{abstract}

\section{INTRODUCTION}

Brownian oscillator is the simplest and exactly solvable system in quantum dissipation theory. ${ }^{1-4}$ Physically it serves as an elementary model in diversified fields, such as quantum optics, quantum statistics, spectroscopies, and chemical dynamics in condensed phases. ${ }^{5-14}$ In this model, the harmonic oscillator $(q)$ is coupled to a heat bath of harmonic oscillators $\left(\left\{x_{j}\right\}\right)$ in a bilinear form. The total composite material Hamiltonian reads ${ }^{2-4}$

$$
H_{\mathrm{M}}=H_{0}(p, q)+\sum_{j}\left[\frac{p_{j}^{2}}{2 m_{j}}+\frac{1}{2} m_{j} \omega_{j}^{2}\left(x_{j}-\frac{c_{j} q}{m_{j} \omega_{j}^{2}}\right)^{2}\right],
$$

with

$$
H_{0}=\frac{p^{2}}{2 M}+\frac{1}{2} M \Omega^{2} q^{2} .
$$

The effect of bath is completely characterized by the interaction bath spectral density function: ${ }^{2}$

$$
J(\omega \geq 0)=\frac{\pi}{2} \sum_{j} \frac{c_{j}^{2}}{m_{j} \omega_{j}} \delta\left(\omega-\omega_{j}\right) .
$$

Note that the Caldeira-Leggett model, Eq. (1a), is intrinsically perturbative, as the bath is assumed to be harmonic, i.e., $h_{\mathrm{B}}=\sum_{j}\left[p_{j}^{2} /\left(2 m_{j}\right)+m_{j} \omega_{j}^{2} x_{j}^{2} / 2\right]$, and linearly coupled to the system. However, it can be treated exactly without further assumption for an arbitrary system Hamiltonian, including anharmonic cases. ${ }^{2,15-17}$ One can obtain the Feynman-Vernon influence functional path integral expression ${ }^{2,15}$ or its equivalent differential formalism in terms of hierarchical equations of motion ${ }^{16,17}$ for the reduced system dynamics. Great simplification does exist for the Brownian oscillator where the reduced system of Eq. (1b) is also harmonic., ${ }^{2,18-22}$

The dynamics of Brownian oscillators can be studied in terms of the quantum Langevin equation. It starts with the Heisenberg equations of motion for all degrees of freedom, followed by using the formal solutions to the bath ones. The resulting quantum Langevin equation reads $^{2}$

$$
\begin{aligned}
\dot{\hat{p}}(t)= & -M \Omega^{2} \hat{q}(t)-\int_{t_{0}}^{t} \mathrm{~d} \tau \gamma(t-\tau) \hat{p}(\tau)+F\left(t-t_{0}\right) \\
& -M \gamma\left(t-t_{0}\right) \hat{q}\left(t_{0}\right) .
\end{aligned}
$$

Here, $\gamma(t)=M^{-1} \sum_{j}\left(c_{j}^{2} / m_{j} \omega_{j}^{2}\right) \cos \left(\omega_{j} t\right)$ is the friction kernel function; $F(t)=e^{i h_{\mathrm{B}} t / \hbar}\left(\sum_{j} c_{j} x_{j}\right) e^{-i h_{\mathrm{B}} t / \hbar}$ is the stochastic force operator, and its correlation function is related to the bath spectral density and the friction kernel functions via the well-established fluctuation-dissipation theorem. ${ }^{1-3}$ Note that if the initial time is set to be $t_{0} \rightarrow$ $-\infty$, the last term in Eq. (3) is reduced to zero, due to the friction kernel function $\gamma(t \rightarrow \infty)=0$.

The Brownian oscillator system is more often studied in terms of the quantum master equation (QME). The key quantity here is the reduced density operator $\rho(t) \equiv$ $\operatorname{tr}_{\mathrm{B}} \rho_{\mathrm{T}}(t)$, i.e., the bath subspace trace of the total density operator. The exact QME to Brownian oscillators had been constructed via various approaches. These include the calculus-on-path-integral algorithm, ${ }^{18,19}$ the traceover-bath on total-space Wigner-function method, ${ }^{20-22}$ the stochastic field ${ }^{23}$ and the stochastic Schrödinger wave function ${ }^{24}$ approaches. All these cited efforts had not considered the effect of time-dependent external fields.

We have recently constructed an exact QME to a driven Brownian oscillator (DBO) system, with the assumption of initial thermal equilibrium before the timedependent laser field action. ${ }^{3,25}$ In this work, we will revisit the theory with an arbitrary initial factorized state. A single-mode DBO system will be considered for clarity. It can be readily extended to multi-mode systems, together with the applications to some nonlinear spectroscopic problems in condensed phases. ${ }^{7,26,27}$

The remainder of the paper is organized as follows. With the derivation detailed in Appendix A, we present the final results of QME-DBO theory in Sec.II. We discuss its Markovian simplification in Appendix B and its asymptotic equilibrium property in Appendix C. The new feature of the theory is an additional effective field 
correction, resulting from the interplay between external field interaction and non-Markovian dissipation. We analyze the effects of the field correction on the DBO system in Sec. III, and finally conclude the paper in Sec. IV.

\section{THEORY}

\section{A. Model and notations}

The total DBO Hamiltonian in the presence of an external time-dependent field $\epsilon(t)$ reads [cf. Eq. (1)]

$$
H_{\mathrm{T}}(t)=H_{\mathrm{M}}-q \epsilon(t)=H(t)+h_{\mathrm{B}}-q \sum_{j} c_{j} x_{j} .
$$

Here, $H(t)=H_{0}+H_{\text {ren }}-q \epsilon(t)$ is the sum of those in the system space, with the renormalization $H_{\text {ren }}=$ $q^{2} \sum_{j} c_{j}^{2} /\left(2 m_{j} \omega_{j}^{2}\right)=\frac{1}{2} M \gamma(0) q^{2}$. Thus, the system Hamiltonian in Eq. (4) is

$$
H(t)=\frac{p^{2}}{2 M}+\frac{1}{2} M\left[\Omega^{2}+\gamma(0)\right] q^{2}-q \epsilon(t) .
$$

The effects of bath interaction are described by the forceforce correlation function:

$$
C_{\mathrm{B}}(t) \equiv\langle F(t) F(0)\rangle_{\mathrm{B}} \equiv \hbar[a(t)+i b(t)] .
$$

Here, $a(t)$ and $b(t)$ denote the real and imaginary parts of $C_{\mathrm{B}}(t) / \hbar$, respectively; $\langle\cdot\rangle_{\mathrm{B}} \equiv \operatorname{tr}_{\mathrm{B}}\left(\cdot \rho_{\mathrm{B}}^{\mathrm{eq}}\right)$ with $\rho_{\mathrm{B}}^{\mathrm{eq}}=$ $e^{-\beta h_{\mathrm{B}}} / \operatorname{tr}_{\mathrm{B}}\left(e^{-\beta h_{\mathrm{B}}}\right)$ being the bath canonical equilibrium density operator, and $\beta \equiv 1 /\left(k_{\mathrm{B}} T\right)$ the inverse temperature. The imaginary part is the sine transformation of the spectral density (with a factor of $-1 / \pi$ ), and related to the friction kernel function as $b(t)=\frac{M}{2} \dot{\gamma}(t)$. The real part, $a(t)$, depends on temperature and is related to the imaginary part, $b(t)$, via the fluctuation-dissipation theorem. ${ }^{1-3}$

For later use, we denote the response function of the Brownian oscillator system as

$$
\chi(t) \equiv i\langle[\hat{q}(t), \hat{q}(0)]\rangle_{\mathrm{M}} / \hbar .
$$

Here, $\hat{q}(t)=e^{i H_{\mathrm{M}} t / \hbar} q e^{-i H_{\mathrm{M}} t / \hbar}$ and $\langle\cdot\rangle_{\mathrm{M}} \equiv \operatorname{tr}_{\mathrm{M}}\left(\cdot \rho_{\mathrm{M}}^{\mathrm{eq}}\right) \equiv$ $\operatorname{tr}_{\mathrm{M}}\left(\cdot e^{-\beta H_{\mathrm{M}}}\right) / \operatorname{tr}_{\mathrm{M}}\left(e^{-\beta H_{\mathrm{M}}}\right)$, defined in the total system and bath composite material space. From its definition and Eq. (3) (setting $t_{0}=0$ there), we have

$$
\ddot{\chi}(t)+\Omega^{2} \chi(t)+\int_{0}^{t} \mathrm{~d} \tau \gamma(t-\tau) \dot{\chi}(\tau)=0,
$$

with $\chi(0)=0$ and $\dot{\chi}(0)=1 / M$. Let

$$
\hat{\chi}(\omega) \equiv \int_{0}^{\infty} \mathrm{d} t e^{i \omega t} \chi(t), \quad \hat{\gamma}(\omega) \equiv \int_{0}^{\infty} \mathrm{d} t e^{i \omega t} \gamma(t) .
$$

The frequency-domain resolution of Eq. (8) is then

$$
\hat{\chi}(\omega)=\left\{M\left[\Omega^{2}-\omega^{2}-i \omega \hat{\gamma}(\omega)\right]\right\}^{-1} .
$$

Its real and imaginary parts are corresponding to the dispersion and absorption, respectively. The response function $\chi(t)$ will be the key quantity in the following construction of the QME-DBO theory.

\section{B. Quantum master equation: Exact results}

For convenience, we set $t_{0}=0$ hereafter, and assume the total system-bath composite state is initially of an uncorrelated form of $\rho_{\mathrm{T}}(0)=\rho(0) \rho_{\mathrm{B}}^{\text {eq }}$. Due to ergodicity the effects of initial states vanish completely in the course of time and the system relaxes to the thermal equilibrium. In Appendix A we derive the exact QMEDBO theory via the modified Yan-Mukamel method on the Gaussian wave packet (GWP) dynamics. ${ }^{3,25,26}$

The final results are summarized as follows.

$$
\dot{\rho}=-\frac{i}{\hbar}\left[H_{\mathrm{eff}}(t), \rho\right]-\mathcal{R}(t) \rho,
$$

with

$$
H_{\mathrm{eff}}(t)=\frac{p^{2}}{2 M}+\frac{1}{2} M \Omega_{t}^{2} q^{2}-q[\epsilon(t)+\delta \epsilon(t)],
$$

and

$$
\mathcal{R}(t) \rho=\frac{i \Gamma_{t}}{2 \hbar}[q,\{p, \rho\}]+\frac{M \zeta_{t}^{\prime}}{\hbar}[q,[q, \rho]]-\frac{\zeta_{t}}{\hbar}[q,[p, \rho]] .
$$

The involving time-dependent functions are

$$
\begin{aligned}
& \Omega_{t}^{2}=\frac{\ddot{\chi}^{2}(t)-\dddot{\chi}(t) \dot{\chi}(t)}{\dot{\chi}^{2}(t)-\ddot{\chi}(t) \chi(t)}, \\
& \Gamma_{t}=\frac{\dddot{\chi}(t) \chi(t)-\ddot{\chi}(t) \dot{\chi}(t)}{\dot{\chi}^{2}(t)-\ddot{\chi}(t) \chi(t)}, \\
& \zeta_{t}^{\prime}=\int_{0}^{t} \mathrm{~d} \tau \dot{\chi}(\tau) \xi(\tau ; t), \quad \zeta_{t}=\int_{0}^{t} \mathrm{~d} \tau \chi(\tau) \xi(\tau ; t), \\
& \delta \epsilon(t)=\int_{0}^{t} \mathrm{~d} \tau \chi_{\epsilon}(t-\tau ; t) \epsilon(\tau),
\end{aligned}
$$

with

$$
\begin{aligned}
& \xi(\tau ; t) \equiv a(\tau)+\int_{0}^{t} \mathrm{~d} \tau^{\prime} a\left(\tau-\tau^{\prime}\right) \chi_{\epsilon}\left(\tau^{\prime} ; t\right), \\
& \chi_{\epsilon}(\tau ; t) \equiv M\left[\ddot{\chi}(\tau)+\Gamma_{t} \dot{\chi}(\tau)+\Omega_{t}^{2} \chi(\tau)\right] .
\end{aligned}
$$

\section{Comments}

The $H_{\text {eff }}(t)$ of Eq. (11b), even in the absence of $\delta \epsilon(t)$, is neither $H(t)$ [Eq. (5)] nor its Caldeira-Leggett counterpart, $H_{0}-q \epsilon(t)$ [cf. Eq. (1b)]. The latter is recovered in the Markovian limit; see Appendix B. The values of $\chi(0)=\ddot{\chi}(0)=0, \dot{\chi}(0)=1 / M$, and $\dddot{\chi}(0)=$ $-\left[\Omega^{2}+\gamma(0)\right] / M$ lead to $\Omega_{t=0}^{2}=\Omega^{2}+\gamma(0)$ and $\Gamma_{t=0}=0$; i.e., $H_{\text {eff }}(t)$ assumes $H(t)$ [Eq. (5)] at $t=0$ before dissipation occurs. When the solvent fluctuation is much slower than the system response dynamics, Eq. (8) is reduced to $\ddot{\chi}(t)+\left[\Omega^{2}+\gamma(0)\right] \chi(t)=0$; the relaxation is yet to occur. This implies that in the slow modulation limit the bath contributes only to the static reorganization energy.

Temperature enters the QME through $\zeta_{t}^{\prime}$ and $\zeta_{t}$ via the real part of bath correlation function, $a(t) \equiv \operatorname{Re} C_{\mathrm{B}}(t) / \hbar$; 


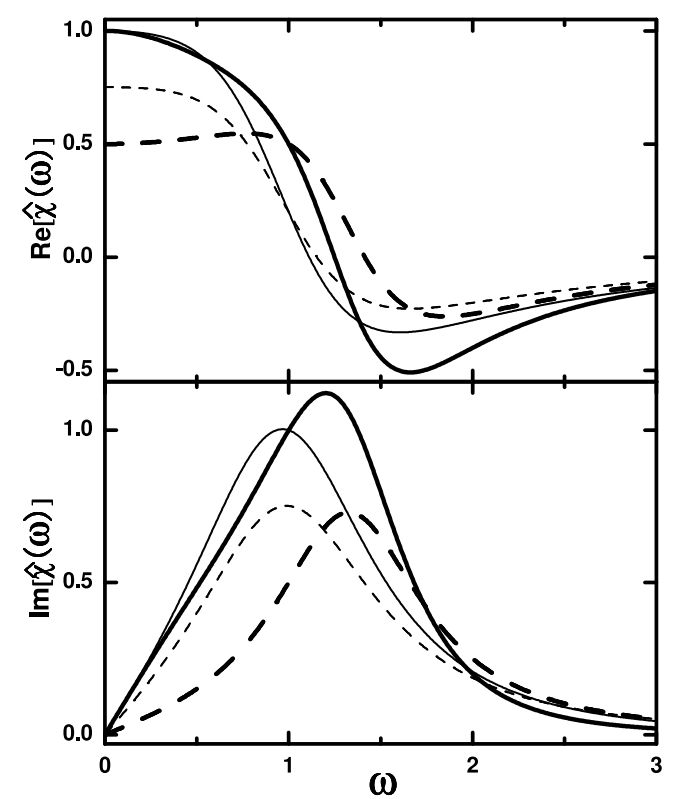

FIG. 1: Real and imaginary parts of the linear response functions $\hat{\chi}(\omega)$ [Eq. (10)], with the coupling bath strength $\Gamma=1$ and two values of spectral width (in unit of $M=\Omega=\hbar=1$ ): $W=2$ (solid) and 5 (thin-solid). The dashed curves are the $\delta \epsilon-$ free approximation counterparts.

cf. Eq. (12c) with Eq. (13a). The asymptotic behaviors of $\zeta_{t}^{\prime}$ and $\zeta_{t}$ are related with the thermal equilibrium variances $\sigma_{p p}^{\text {eq }}$ and $\sigma_{q q}^{\text {eq }}$; see Appendix C. This guarantees the correct equilibrium property of the QME.

The main feature in the present QME-DBO formalism is the inclusion of the effective local-field correction $\delta \epsilon(t)$ [Eqs. (12d) and (13b)]. It results from an interplay between driving and non-Markovian dissipation. In the absence of the external driving field, the above QME formalism is the same as the previous theory; see, for example, Refs. 18 and 19.

\section{DEMONSTRATIONS AND ANALYSIS}

\section{A. The effective field correction}

The effective field correction $\delta \epsilon(t)$ is purely of nonMarkovian nature. (i) It vanishes in the Markovian white noise limit (cf. Appendix B); (ii) For a general nonMarkovian bath, if the field correction $\delta \epsilon$ were neglected, the QME would lead to the system response function $\hat{\chi}(\omega)$ a Markovian form, $\left[M\left(\Omega_{t \rightarrow \infty}^{2}-\omega^{2}-i \omega \Gamma_{t \rightarrow \infty}\right)\right]^{-1}$. The exact result of Eq. (10) can only be recovered with the inclusion of $\delta \epsilon(t)$ in the exact QME-DBO theory; (iii) $\delta \epsilon(t)$ is most important in the low frequency driving and intermediate bath memory region; (iv) The effect of $\delta \epsilon(t)$ is cooperative, leading to the enhancement of polarization.

Note that the asymptotic limit throughout this pa-

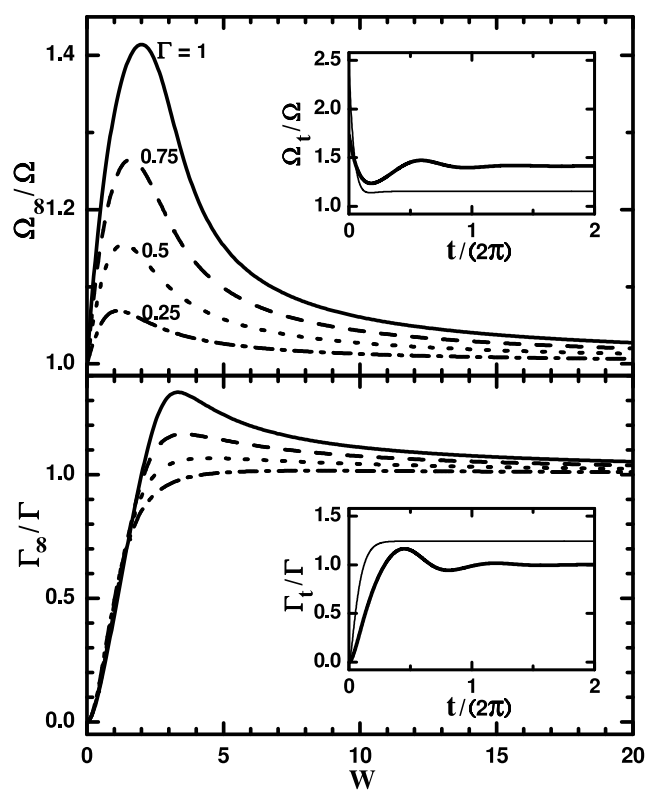

FIG. 2: The asymptotic $\Omega_{t \rightarrow \infty}$ and $\Gamma_{t \rightarrow \infty}$ as functions of $W$ for $\Gamma=1$ (solid), 0.75 (dash), 0.5 (dot), and 0.25 (dash-dot), respectively. Shown in the insets are the transient $\Omega_{t}$ and $\Gamma_{t}$ as functions of time, with the coupling bath strength $\Gamma=1$ and two values of spectral width, $W=2$ (solid) and 5 (thinsolid). All are in unit of $M=\Omega=\hbar=1$.

per refers to the $t>t_{c}$ regime, where $t_{c}$ denotes the characteristic time of the system response function having $\chi\left(t>t_{c}\right) \approx 0$. We adopt hereafter the internal unit of $M=\Omega=\hbar=k_{\mathrm{B}}=1$, and the Drude bath model, $\gamma(t \geq 0)=\Gamma W e^{-W t}$, for the purpose of numerical demonstrations, where $\Gamma$ and $W$ denote the bath coupling strength and spectral width, respectively.

Figure 1 compares the system response functions evaluated with and without $\delta \epsilon(t)$. The difference between them is considerable in the intermediate bath memory region, but vanishes in both $W \ll \Omega$ (narrow bandwidth) and $W \gg \Omega$ (Markovian) limits (thus not shown).

Before further demonstrations, let us examine $\Omega_{t}$ and $\Gamma_{t}$, as these two quantities are involved in $\delta \epsilon(t)$ [cf. Eq. (13b)], as well as in the Brownian oscillator part of the QME. Their initial values are $\Omega_{t=0}=\sqrt{\Omega^{2}+\gamma(0)}$ and $\Gamma_{t=0}=0$, as discussed in Sec. II C. Figure 2 depicts the asymptotic values of $\Omega_{t \rightarrow \infty}$ and $\Gamma_{t \rightarrow \infty}$ varying with the bath spectral width $W$ and coupling strength $\Gamma$, together with the insets showing $\Omega_{t}$ and $\Gamma_{t}$ as functions of time at $\Gamma=1, W=2$ and 5 . The oscillations of $\Omega_{t}$ and $\Gamma_{t}$ vanish at large $W$. The oscillatory frequency is about that of the system response function. For the asymptotic behavior, $\Gamma_{\infty} \rightarrow 0$ when $W \ll \Omega$ and $\Gamma_{\infty} \rightarrow \Gamma$ when $W \gg \Omega$, while $\Omega_{\infty} \rightarrow \Omega$ in both limits.

Let us now turn to the local-field correction, $\delta \epsilon(t)$ of Eq. (12d). Note that $\delta \epsilon$ depends linearly on the external driving field $\epsilon(t)$. We may therefore analyze the effect of the field correction with a continuous-wave (cw) drive, 
$\epsilon(t)=\epsilon_{0} \sin \left(\omega_{f} t\right)$, as an arbitrary field is composed of $c w$ fields.

Note that the continuous-wave driven Brownian motion had been widely studied on two-state/double-well systems, ${ }^{28,29}$ Rydberg atoms in a waveguide, ${ }^{30}$ as well as dissipative harmonic oscillators, ${ }^{31,32}$ with both linear and quadratic dipole moments. Although the additive term of an effect driving (that would lead to a form of $\delta \epsilon)$ was mentioned, see for example, the Appendix B of Ref. 32, its influence was not studied. And with the further Markovian approximation made there, the effective field correction vanishes and goes unnoticed, as consistent with the limiting case of the present work; see Fig. 3 below and the discussion follows.

The asymptotic behavior of $\delta \epsilon(t)$ associating with the $c w$-field can be obtained as [cf. Eqs. (12d) and (13b) and noting that $\left.\hat{\chi}^{*}(\omega)=\hat{\chi}(-\omega)\right]$

$$
\delta \epsilon(t) \rightarrow \epsilon_{0} \operatorname{Im}\left[e^{i \omega_{f} t} \hat{\chi}_{\epsilon}^{*}\left(\omega_{f}\right)\right]=r \epsilon_{0} \sin \left(\omega_{f} t-\theta\right),
$$

with

$$
\hat{\chi}_{\epsilon}\left(\omega_{f}\right) \equiv \int_{0}^{\infty} \mathrm{d} \tau e^{i \omega_{f} \tau} \chi_{\epsilon}(\tau ; t \rightarrow \infty) \equiv r e^{i \theta} .
$$

In other words, $\hat{\chi}_{\epsilon}\left(\omega_{f}\right)$ defines the relative magnitude $r$ and the retarded phase $\theta$ of the asymptotic $\delta \epsilon(t)$ with respective to the original $c w$ driving field. Figure 3 demonstrates the $r$ and $\theta$, for their dependence on the bath spectral width $W$, the coupling strength $\Gamma$, and the $c w-$ field frequency $\omega_{f}$.

The relative magnitude $r$ of $\delta \epsilon_{t \rightarrow \infty}$, as shown in the upper panel of Fig. 3, exhibits turnover behaviors, as function of bath spectral width $W$. On the other hand, it decreases monotonically as the external $c w$-field frequency $\omega_{f}$ increases (see the inset of upper panel), due to the cancellation for the highly oscillatory $\epsilon(t)$. As the function $r(W)$ is concerned, both its peak and width increase rather rapidly as the system-bath coupling strength $\Gamma$ increases. We may refer the case being of experimental significance if $r=\left|\delta \epsilon / \epsilon_{0}\right| \geq 0.2$. With the moderately strong value of $\Gamma=1$, the field correction would take a significant effect within $0.6 \Omega \leq W \leq 7 \Omega$, which often covers the experimentally important region.

The retarded phase $\theta$ of $\delta \epsilon_{t \rightarrow \infty}$, as shown in the lower panel of Fig. 3, decreases from $\pi / 2$ at long memory $(W \ll \Omega)$ regime, to 0 in the Markovian $(W \gg \Omega)$ limit; meanwhile it increases from 0 to $\pi / 2$ as the driving field frequency increases; see the inset in the lower panel. These observations are consistent with the picture that the retarded phase reflects the relative bath response time with respect to the inverse frequency of driving.

We have thus clearly demonstrated that the effective field correction $\delta \epsilon(t)$ results from the interplay between the driving and non-Markovian dissipation. Remarkably, the observed retarded phases are all of $\theta \in[0, \pi / 2]$. Thus, the effective field correction is of cooperative effect that enhances the influence of driving. The physical origin of

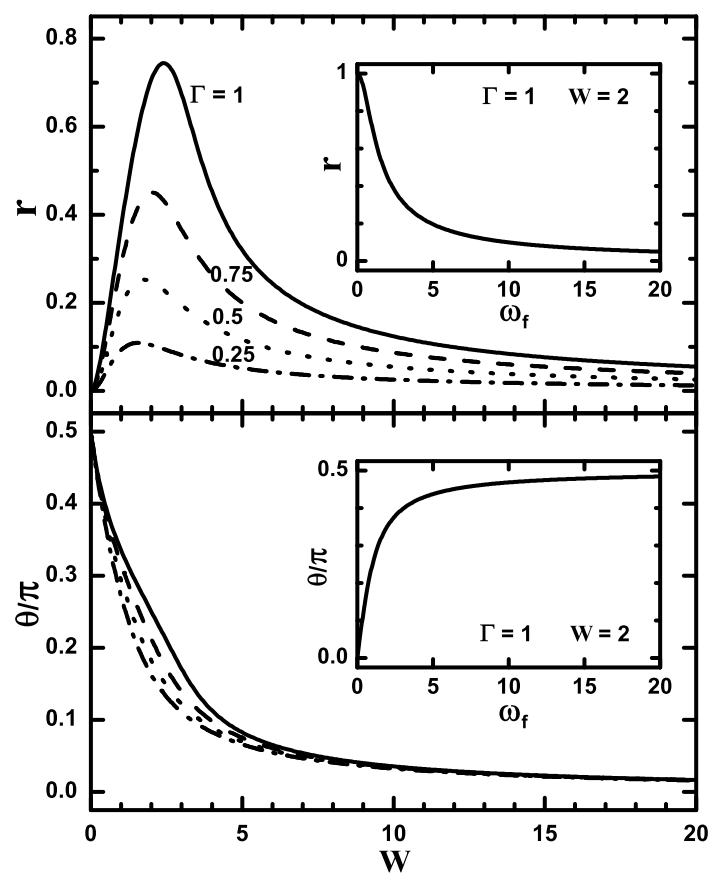

FIG. 3: The amplitude scaling factor $r$ (upper) and retarded phase $\theta$ (lower), which characterize the asymptotic $\delta \epsilon(t) \rightarrow r \epsilon_{0} \sin \left(\omega_{f} t-\theta\right)$, as functions of $W$, with $\omega_{f}=1$ and $\Gamma=1$ (solid), 0.75 (dash), 0.5 (dot), and 0.25 (dash-dot), respectively. Shown in the insets are $r$ and $\theta$ as functions of the $c w$ driving field frequency $\omega_{f}$, evaluated with $\Gamma=1$ and $W=2$. All are in unit of $M=\Omega=\hbar=1$.

this enhancement is due to the bath-induced disperse of $\delta q$ that leads to an effective larger polarization. Neglect of $\delta \epsilon$ will underestimate the influence of driving such as the field-induced polarizations to be demonstrated below.

\section{B. Driven Brownian oscillator dynamics}

Consider now the dynamics of system in the presence of an external field. Let us start with the asymptotic behaviors under the $c w$-field drive. The mean values of the coordinate and momentum can be obtained as [cf. Eq. (A1)]

$$
\begin{aligned}
& q_{t} \longrightarrow \epsilon_{0} \operatorname{Im}\left[e^{i \omega_{f} t} \hat{\chi}^{*}\left(\omega_{f}\right)\right] \\
& p_{t} \longrightarrow \epsilon_{0} M \omega_{f} \operatorname{Re}\left[e^{i \omega_{f} t} \hat{\chi}^{*}\left(\omega_{f}\right)\right]
\end{aligned}
$$

The system energy, in terms of $\left\langle H_{0}\right\rangle=\frac{1}{2 M}\left\langle p^{2}\right\rangle+$ $\frac{1}{2} M \Omega^{2}\left\langle q^{2}\right\rangle$, has the asymptotic expression of

$$
\begin{aligned}
\left\langle H_{0}\right\rangle \longrightarrow & \left(\frac{\sigma_{p p}^{\mathrm{eq}}}{2 M}+\frac{1}{2} M \Omega^{2} \sigma_{q q}^{\mathrm{eq}}\right)+\frac{1}{2} M \Omega^{2} \epsilon_{0}^{2}\left|\hat{\chi}\left(\omega_{f}\right)\right|^{2} \\
& +\frac{1}{2} M\left(\omega_{f}^{2}-\Omega^{2}\right) \epsilon_{0}^{2}\left\{\operatorname{Re}\left[e^{i \omega_{f} t} \hat{\chi}^{*}\left(\omega_{f}\right)\right]\right\}^{2} .
\end{aligned}
$$

The three terms in the right-hand-side of the above equation describe, respectively, the thermal average 


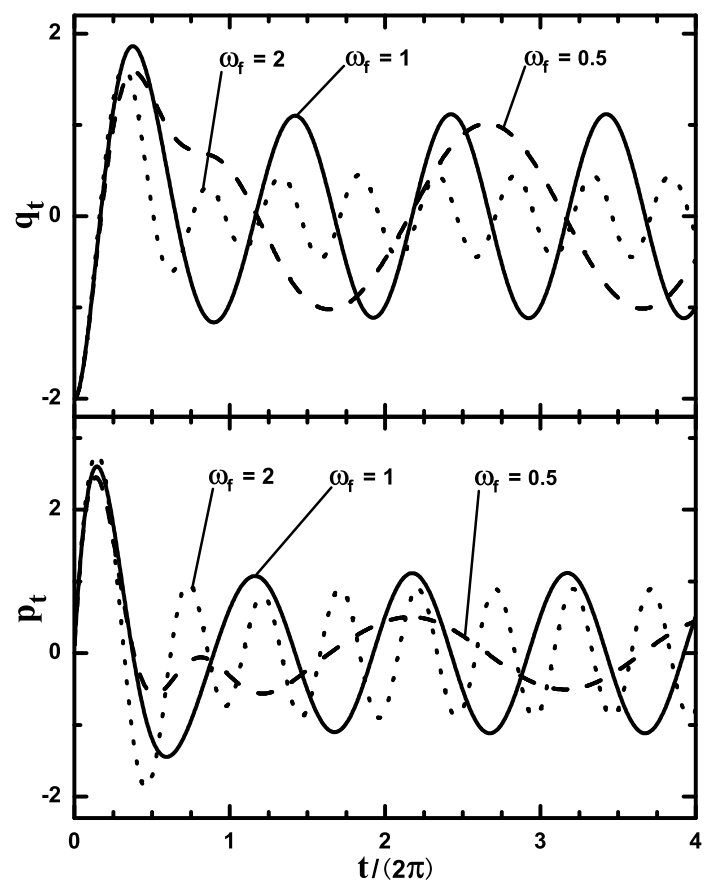

FIG. 4: The evolution of $q_{t}$ (upper) and $p_{t}$ (lower) under the $c w$-field $\epsilon(t)=\epsilon_{0} \sin \left(\omega_{f} t\right)$, with $\epsilon_{0}=1$ and $\omega_{f}=1$ (solid), 0.5 (dash), and 2 (dot), respectively. The bath parameters are $\Gamma=1$ and $W=2$. All are in unit of $M=\Omega=\hbar=1$.

value, the $c w$-field induced static polarization with the system elongation of $\delta q=\left|\epsilon_{0} \hat{\chi}\left(\omega_{f}\right)\right|$, and the residue oscillatory breathing. In the absence of dissipation, $\hat{\chi}(\omega)=\left[M\left(\Omega^{2}-\omega^{2}\right)\right]^{-1}$, both the static polarization and the oscillatory breathing terms diverge at $\omega_{f}=\Omega$. This is consistent with the classical energy climbing picture of a $c w$-field driven oscillator. In the presence of dissipation, the oscillatory breathing is asymptotically quenched at resonance, $\omega_{f}=\Omega$, while the static polarization contribution remains finite. Both the fieldinduced energy climbing and breathing into and out of the system are compensated by dissipation. It is also noticed that the resonance condition is closely related to the choice of reduced system Hamiltonian. For example, if the reduced system energy is examined in terms of $\langle H(t=0)\rangle=\frac{1}{2 M}\left\langle p^{2}\right\rangle+\frac{1}{2} M \Omega_{t=0}^{2}\left\langle q^{2}\right\rangle$, similar expression of Eq. (16) holds, but with the resonance condition being replaced by $\omega_{f}=\Omega_{t=0}=\sqrt{\Omega^{2}+\gamma(0)}$.

Demonstrated below will be the time evolution of DBO systems, with both a $c w$-field (cf. Figs. 4-6) and a halfcycle of the field (cf. Fig. 7). The results will be reported in terms of the first- and second-order cumulants, $\left\{q_{t}, p_{t}\right\}$ and $\left\{\sigma_{q q}(t), \sigma_{p q}(t), \sigma_{p p}(t)\right\}$, that fully characterize the GWP of the DBO system (cf. Appendix A), and also in terms of $\left\langle H_{0}\right\rangle$ or polarization that is experimentally observable. Note that $\left\{q_{t}, p_{t}\right\}$ do not depend on temperature, while $\left\{\sigma_{q q}(t), \sigma_{p q}(t), \sigma_{p p}(t)\right\}$ do not depend on the driving fields; and their equations of motion, Eq. (A6) and Eq. (A8), are independent.

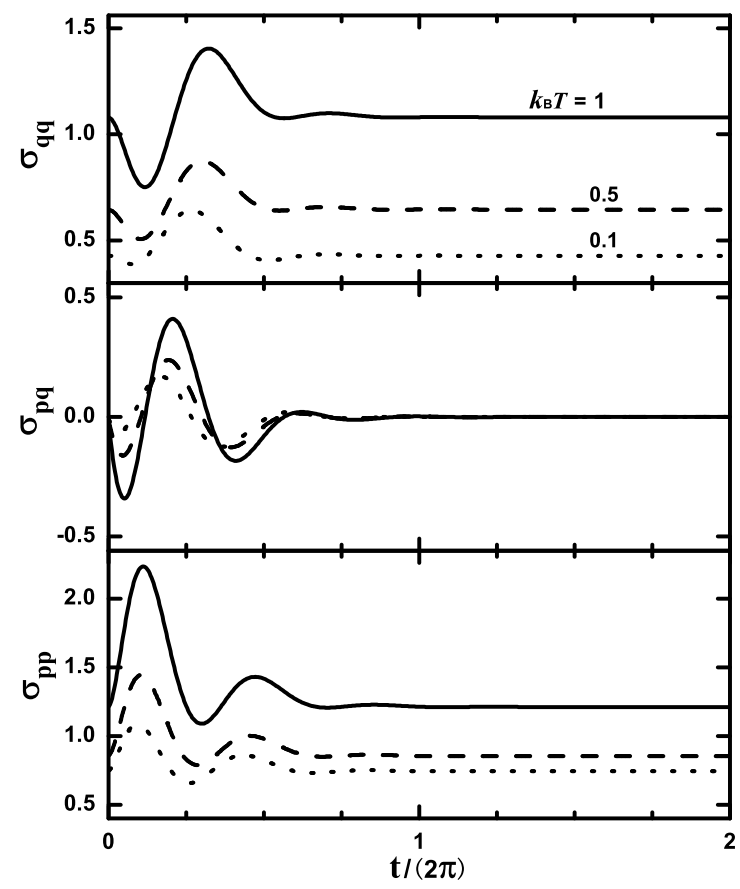

FIG. 5: The evolution of the phase-space variances, $\sigma_{q q}(t)$, $\sigma_{p q}(t)$, and $\sigma_{p p}(t)$, at temperature $T=1$ (solid), 0.5 (dash), and 0.1 (dot). The bath parameters are $\Gamma=1$ and $W=2$. All are in unit of $M=\Omega=\hbar=k_{\mathrm{B}}=1$.

For the DBO system in the presence of a $c w$-field $\epsilon(t>0)=\epsilon_{0} \sin \left(\omega_{f} t\right)$, we set the initial GWP to have the first-order cumulants of $\left\{q_{t=0}, p_{t=0}\right\}=\{-2,0\}$ and the second-order cumulants the same as their thermal equilibrium values. This mimics the scenario of ultra-fast $\mathrm{UV} /$ visible laser pulse excitation of an electronic ground state Gaussian wave packet onto a linearly displaced excited surface, immediately followed by the $c w$ infrared field. The wave packet dynamics on the excited surface under the influence of the $c w$-field is then studied.

Figures 4 depicts the evolution of $q_{t}$ and $p_{t}$. Their asymptotic behaviors, as described by Eq. (15), oscillate with the $c w$-field frequency $\omega_{f}$.

Figures 5 demonstrates the evolution of $\sigma_{q q}, \sigma_{p q}$, and $\sigma_{p p}$. Although they were initially of the equilibrium values and they are independent of the external field afterward, the second-order cumulants do oscillate and take time back to equilibrium. This is because there was no excited state wave packet initially and the dissipation considered here only starts when there is a real occupation upon excitation. Therefore the variances of the pumped GWP, as depicted in Fig. 5, do experience $d y$ namics solvation.

Figure 6 shows the evolution of the system energy, $\left\langle H_{0}\right\rangle=\frac{1}{2 M}\left\langle p^{2}\right\rangle+\frac{1}{2} M \Omega^{2}\left\langle q^{2}\right\rangle$. The asymptotic behavior had been described in Eq. (16) and analyzed thereafter. In the non-resonance condition, $\omega_{f} \neq \Omega$, the energy flows in and out with the $c w$-field frequency $\omega_{f}$. This energy breathing is quenched at resonance, $\omega_{f}=\Omega$. Shown in the dashed curves are the $\delta \epsilon$-free counterparts 


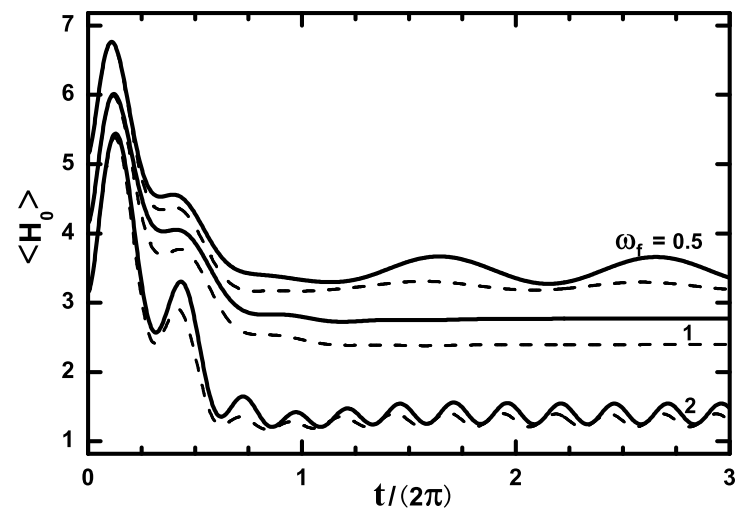

FIG. 6: The evolution of the mean energy $\left\langle H_{0}\right\rangle$, driven by the $c w$-field with $\epsilon_{0}=1$ and $\omega_{f}=0.5$ (shifted by +2 ), 1 (shifted by +1 ), and 2 (no shift). The bath parameters are $\Gamma=1$ and $W=2$ at temperature $T=1$. The dashed curves are the $\delta \epsilon$-free approximation counterparts. All are in unit of $M=\Omega=\hbar=k_{\mathrm{B}}=1$.

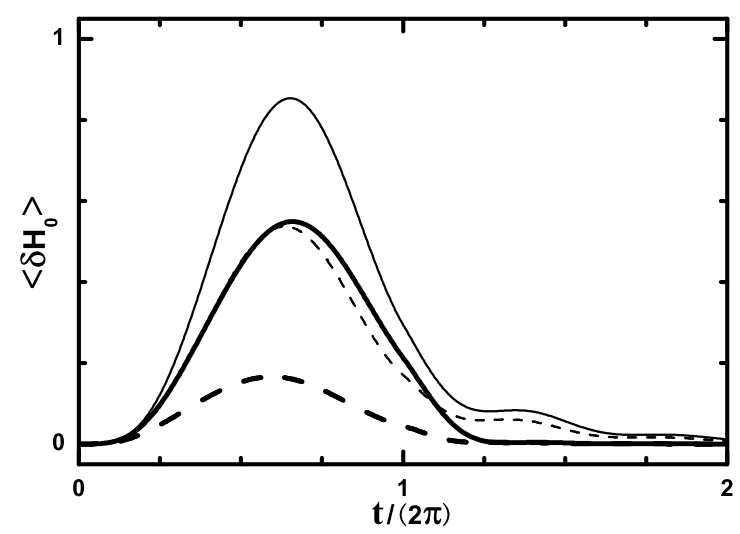

FIG. 7: The evolution of $\left\langle\delta H_{0}\right\rangle=\left\langle H_{0}\right\rangle-\left\langle H_{0}\right\rangle_{\text {eq }}$, driven by a half cycle of the $c w$-field with $\epsilon_{0}=1$ and $\omega_{f}=0.5$ on an initial thermal equilibrium state at temperature $T=1$. The bath parameters are $W=2$, and $\Gamma=1$ (solid) and 0.5 (thin-solid), respectively. The dashed curves are the $\delta \epsilon-$ free approximation counterparts. All are in unit of $M=\Omega=\hbar=k_{\mathrm{B}}=1$.

for comparison. Their difference is proportional to the field strength, and also depends on the difference between the exact $\hat{\chi}\left(\omega_{f}\right)$ and its approximation; cf. Eq. (16) and Fig. 1.

Consider now the DBO dynamics driven by a halfcycle of the sine-function field. The system is assumed to be initially at the thermal equilibrium. Figure 7 reports the resulting dynamics in terms of the polarization/magnetization related quantity, $\left\langle\delta H_{0}\right\rangle=\left\langle H_{0}\right\rangle-$ $\left\langle H_{0}\right\rangle_{\text {eq. }}$. Again it shows that the effect of $\delta \epsilon$ is cooperative, and the neglect of this correlated driving and non-Markovian dissipation contribution will significantly underestimate the field-induced polarization.

\section{SUMMARY}

This work presents the exact QME-DBO theory, for which the time-dependent external field interaction is considered explicitly. It shows explicitly that the correlated driving and dissipation result in an effective field correction that enhances the polarization. This cooperative property is intrinsically non-Markovian and reflects the effective bath response to external field applied to the system. It vanishes in both the Markovian and slow modulation bath limits, but it is important in the intermediate bath memory and low-frequency driving region.

For the DBO system, the correlated effect of driving and non-Markovian dissipation only appears as a field correction on the coherent motion. In a general anharmonic system, this correlated effect is expected to have much rich implications on the dissipative dynamics even in the weak system-bath coupling regime, see for example, Refs. 33 and 34. The exact non-Markovian dissipative dynamics of general systems under arbitrary external fields can be studied via the hierarchical equations of motion approach. ${ }^{17,35-39}$ These include the quantum transport systems, such as the transient current under the time-dependent bias voltage reported recently. ${ }^{40,41}$

\section{Acknowledgments}

Support from the National Natural Science Foundation of China (20533060 and 20773114), Ministry of Education of China (NCET-05-0546), National Basic Research Program of China (2006CB922004), and RGC Hong Kong is acknowledged.

\section{APPENDIX A: THE WIGNER PHASE-SPACE GAUSSIAN WAVE PACKET APPROACH}

The QME-DBO theory [Eqs. (11) to (13)] can be derived via the modified Yan-Mukamel method on the Gaussian wave packet (GWP) dynamics as follows. ${ }^{3,25,26}$ The solution to the Langevin equation of the DBO system reads ${ }^{3,25}$

$$
\begin{aligned}
{\left[\begin{array}{c}
\hat{q}(t) \\
\hat{p}(t)
\end{array}\right]=} & \mathbf{T}(t)\left[\begin{array}{l}
\hat{q}(0) \\
\hat{p}(0)
\end{array}\right] \\
& +\int_{0}^{t} \mathrm{~d} \tau \mathbf{T}(t-\tau)\left[\begin{array}{c}
0 \\
\epsilon(\tau)+F(\tau)
\end{array}\right],
\end{aligned}
$$

with $\mathbf{T}(t)$ a $2 \times 2$ matrix:

$$
\mathbf{T}(t) \equiv\left[\begin{array}{cc}
M \dot{\chi}(t) & \chi(t) \\
M^{2} \ddot{\chi}(t) & M \dot{\chi}(t)
\end{array}\right] .
$$

The Langevin equation in the time-local prescription is then

$$
\left[\begin{array}{c}
\dot{\hat{q}}(t) \\
\dot{\hat{p}}(t)
\end{array}\right]=\boldsymbol{\Lambda}(t)\left[\begin{array}{c}
\hat{q}(t) \\
\hat{p}(t)
\end{array}\right]+\left[\begin{array}{c}
0 \\
\epsilon_{\mathrm{eff}}(t)+F_{\mathrm{eff}}(t)
\end{array}\right] .
$$


Here, $\epsilon_{\mathrm{eff}}(t) \equiv \epsilon(t)+\delta \epsilon(t)$ and

$$
\begin{aligned}
& F_{\text {eff }}(t) \equiv F(t)+\int_{0}^{t} \mathrm{~d} \tau \chi_{\epsilon}(t-\tau ; t) F(\tau), \\
& \boldsymbol{\Lambda}(t)=\dot{\mathbf{T}}(t) \mathbf{T}^{-1}(t)=\left[\begin{array}{cc}
0 & 1 / M \\
-M \Omega_{t}^{2} & -\Gamma_{t}
\end{array}\right],
\end{aligned}
$$

with $\Omega_{t}^{2}, \Gamma_{t}$, and $\delta \epsilon(t)$ being given in Eqs. (12).

We can then obtain the equations of motion for all the cumulants of GWP: $:^{3,25}$

$$
\begin{aligned}
{\left[\begin{array}{c}
\dot{q}_{t} \\
\dot{p}_{t}
\end{array}\right] } & =\boldsymbol{\Lambda}(t)\left[\begin{array}{c}
q_{t} \\
p_{t}
\end{array}\right]+\left[\begin{array}{c}
0 \\
\epsilon_{\mathrm{eff}}(t)
\end{array}\right], \\
\dot{\mathbf{W}}_{t} & =\boldsymbol{\Lambda}(t) \mathbf{W}_{t}+\mathbf{W}_{t} \boldsymbol{\Lambda}^{T}(t) \\
& +\operatorname{Re}\left[\begin{array}{cc}
0 & \left\langle F_{\text {eff }}(t) \delta \hat{q}(t)\right\rangle_{0} \\
\left\langle F_{\text {eff }}(t) \delta \hat{q}(t)\right\rangle_{0} & 2\left\langle F_{\text {eff }}(t) \delta \hat{p}(t)\right\rangle_{0}
\end{array}\right] .
\end{aligned}
$$

Here, $\mathbf{W}_{t} \equiv\left[\begin{array}{cc}\sigma_{q q(t)} & \sigma_{p q(t)} \\ \sigma_{p q}(t) & \sigma_{p p(t)}\end{array}\right]$ denotes the phase-space variances of GWP, $\delta \hat{q}(t) \equiv \hat{q}(t)-q_{t}, \delta \hat{p}(t) \equiv \hat{p}(t)-p_{t}$, and $\langle\cdot\rangle_{0} \equiv \operatorname{tr}_{\mathrm{M}}\left[\cdot \rho_{\mathrm{T}}(0)\right]$. We also use the initial factorized state $\rho_{\mathrm{T}}(0)=\rho(0) \rho_{\mathrm{B}}^{\text {eq }}$. From Eqs. (A1) and (A4), we have

$$
\begin{aligned}
\left\langle F_{\text {eff }}(t)\left[\begin{array}{l}
\delta \hat{q}(t) \\
\delta \hat{p}(t)
\end{array}\right]\right\rangle_{0}= & \int_{0}^{t} \mathrm{~d} \tau\left[\begin{array}{c}
\chi(t-\tau) \\
M \dot{\chi}(t-\tau)
\end{array}\right]\left\{C_{\mathrm{B}}(t-\tau)\right. \\
& \left.+\int_{0}^{t} \mathrm{~d} \tau^{\prime} \chi_{\epsilon}\left(t-\tau^{\prime} ; t\right) C_{\mathrm{B}}\left(\tau^{\prime}-\tau\right)\right\} .
\end{aligned}
$$

Here, $C_{\mathrm{B}}(t)$ is the bath correlation function of Eq. (6). Comparing with Eqs. (12c) and (13a), it is easy to find that $\operatorname{Re}\left\langle F_{\text {eff }}(t) \delta \hat{q}(t)\right\rangle_{0}=\hbar \zeta_{t}$ and $\operatorname{Re}\left\langle F_{\text {eff }}(t) \delta \hat{p}(t)\right\rangle_{0}=$ $M \hbar \zeta_{t}^{\prime}$. Substituting those into Eq. (A7), we obtain

$$
\dot{\mathbf{W}}_{t}=\boldsymbol{\Lambda}(t) \mathbf{W}_{t}+\mathbf{W}_{t} \boldsymbol{\Lambda}^{T}(t)+\hbar\left[\begin{array}{cc}
0 & \zeta_{t} \\
\zeta_{t} & 2 M \zeta_{t}^{\prime}
\end{array}\right] .
$$

Now using the standard Yan-Mukamel method, ${ }^{26}$ we arrive at the QME-DBO in the Wigner representation:

$$
\begin{aligned}
\partial_{t} \rho_{\mathrm{W}}= & {\left[-\frac{\partial}{\partial q} \frac{p}{M}+M \Omega_{t}^{2} \frac{\partial}{\partial p} q-\epsilon_{\mathrm{eff}}(t) \frac{\partial}{\partial p}\right] \rho_{\mathrm{W}} } \\
& +\left[\Gamma_{t} \frac{\partial}{\partial p} p+M \hbar \zeta_{t}^{\prime} \frac{\partial^{2}}{\partial p^{2}}+\hbar \zeta_{t} \frac{\partial^{2}}{\partial p \partial q}\right] \rho_{\mathrm{W}} .
\end{aligned}
$$

This is equivalent to Eq. (11).

\section{APPENDIX B: THE MARKOVIAN LIMIT}

In the Markovian white noise limit, $\gamma(t)=2 \Gamma \delta(t)$ or $\hat{\gamma}(\omega)=\Gamma$, the system response function [Eq. (10)] reduces to

$$
\chi(t \geq 0)=(M \tilde{\Omega})^{-1} \sin (\tilde{\Omega} t) e^{-\Gamma t / 2},
$$

where $\tilde{\Omega} \equiv\left(\Omega^{2}-\Gamma^{2} / 4\right)^{1 / 2}$. It results in

$$
\Omega_{t}=\Omega, \quad \Gamma_{t}=\Gamma, \quad \chi_{\epsilon}(\tau ; t)=0 .
$$

Thus the effective field correction [Eq. (12d)] vanishes in this limit. It is well known that $\sigma_{p p}^{\mathrm{eq}}$ diverges in the Markovian white noise limit without further the high-temperature assumption. ${ }^{1,2}$ Same situation applies also to $\zeta_{t}$ and $\zeta_{t}^{\prime}$ [cf. Eq. (C1)]. We have therefore the high-temperature limit of $a(t)=M k_{\mathrm{B}} T \gamma(t) / \hbar$, so that $\zeta_{t}^{\prime}=\Gamma k_{\mathrm{B}} T / \hbar$ and $\zeta_{t}=0$. Thus, Eq. (11) is reduced to

$$
\begin{aligned}
\dot{\rho}(t)= & -\frac{i}{\hbar}\left[H_{0}-q \epsilon(t), \rho(t)\right]-\frac{i \Gamma}{2 \hbar}[q,\{p, \rho(t)\}] \\
& -\frac{M \Gamma k_{\mathrm{B}} T}{\hbar^{2}}[q,[q, \rho(t)]] .
\end{aligned}
$$

This is the Caldeira-Leggett equation, ${ }^{4}$ which has also been extended to anharmonic systems with an arbitrary system-bath coupling mode $Q$ as $^{42,43}$

$$
\begin{aligned}
\dot{\rho}(t)= & -\frac{i}{\hbar}\left[H_{0}-\hat{\mu} \epsilon(t), \rho(t)\right]+\frac{\Gamma}{2 \hbar^{2}}\left[Q,\left\{\left[H_{0}, Q\right], \rho(t)\right\}\right] \\
& -\frac{\Gamma k_{B} T}{\hbar^{2}}[Q,[Q, \rho(t)]] .
\end{aligned}
$$

Note that the system Hamiltonian involved here is $H_{0}$ in the Caldeira-Leggett form.

\section{APPENDIX C: EQUILIBRIUM PROPERTY}

In this appendix we will show that $\zeta_{t}^{\prime}$ and $\zeta_{t}$ have the following asymptotic expressions,

$$
\hbar \zeta_{t}^{\prime} \stackrel{t>t_{f}}{\longrightarrow} \Gamma_{t} \sigma_{p p}^{\mathrm{eq}} / M, \quad \hbar \zeta_{t} \stackrel{t>t_{\digamma}}{\longrightarrow} M \Omega_{t}^{2} \sigma_{q q}^{\mathrm{eq}}-\sigma_{p p}^{\mathrm{eq}} / M .
$$

Here, $t_{c}$ is the characteristic time of the Brownian response function such that $\chi\left(t>t_{c}\right) \approx 0$. Thus, Eq. (A8) behaves asymptotically as

$$
\dot{\mathbf{W}}_{t} \stackrel{t>t_{\mathrm{f}}}{\longrightarrow} \boldsymbol{\Lambda}(t)\left(\mathbf{W}_{t}-\mathbf{W}_{\mathrm{eq}}\right)+\left(\mathbf{W}_{t}-\mathbf{W}_{\mathrm{eq}}\right) \boldsymbol{\Lambda}^{T}(t) .
$$

This guarantees the correct equilibrium property of QME. The involving equilibrium phase-space variances are $\sigma_{p q}^{\mathrm{eq}}=0$ and $^{1-3}$

$$
\begin{aligned}
\sigma_{q q}^{\mathrm{eq}} & =\frac{\hbar}{\pi} \int_{-\infty}^{\infty} \mathrm{d} \omega \frac{\operatorname{Im}[\hat{\chi}(\omega)]}{1-e^{-\beta \hbar \omega}}, \\
\sigma_{p p}^{\mathrm{eq}} & =\frac{M^{2} \hbar}{\pi} \int_{-\infty}^{\infty} \mathrm{d} \omega \frac{\omega^{2} \operatorname{Im}[\hat{\chi}(\omega)]}{1-e^{-\beta \hbar \omega}} .
\end{aligned}
$$

To prove Eq. (C1), let us recast Eq. (12c) with Eqs. (13) 
as follows.

$$
\begin{aligned}
\zeta_{t}= & M\left[\dot{\chi}(t)+\Gamma_{t} \chi(t)\right] \int_{0}^{t} \mathrm{~d} \tau a(t-\tau) \chi(\tau) \\
& +M \chi(t) \int_{0}^{t} \mathrm{~d} \tau a(t-\tau) \dot{\chi}(\tau) \\
& -M \int_{0}^{t} \mathrm{~d} \tau \int_{0}^{t} \mathrm{~d} \tau^{\prime} \dot{\chi}(\tau) a\left(\tau-\tau^{\prime}\right) \dot{\chi}\left(\tau^{\prime}\right) \\
& +M \Omega_{t}^{2} \int_{0}^{t} \mathrm{~d} \tau \int_{0}^{t} \mathrm{~d} \tau^{\prime} \chi(\tau) a\left(\tau-\tau^{\prime}\right) \chi\left(\tau^{\prime}\right), \\
\zeta_{t}^{\prime}= & M \Omega_{t}^{2} \chi(t) \int_{0}^{t} \mathrm{~d} \tau a(t-\tau) \chi(\tau) \\
& +M \dot{\chi}(t) \int_{0}^{t} \mathrm{~d} \tau a(t-\tau) \dot{\chi}(\tau) \\
& +M \Gamma_{t} \int_{0}^{t} \mathrm{~d} \tau \int_{0}^{t} \mathrm{~d} \tau^{\prime} \dot{\chi}(\tau) a\left(\tau-\tau^{\prime}\right) \dot{\chi}\left(\tau^{\prime}\right) .
\end{aligned}
$$

Note that $a(t)=\operatorname{Re}\left[C_{\mathrm{B}}(t)\right] / \hbar$ is an even function and related to the bath spectral density via the fluctuationdissipation theorem: $:^{2,3}$

$$
a(t)=\frac{1}{2 \pi} \int_{-\infty}^{\infty} \mathrm{d} \omega \frac{J(\omega)}{1-e^{-\beta \hbar \omega}}\left(e^{i \omega t}+e^{-i \omega t}\right)
$$

Consider now the $t>t_{c}$ regime where $\chi\left(t>t_{c}\right) \approx$ 0 , the first two terms of both Eqs. (C4a) and (C4b) can be neglected, and every upper limit of the integrations can be set to $\infty$. Then substituting Eq.(C5) into Eq. (C4), performing Fourier transforms, and using the identity $\operatorname{Im}[\hat{\chi}(\omega)]=J(\omega)|\hat{\chi}(\omega)|^{2}$ with noticing $J(\omega)=M \omega \operatorname{Re}[\hat{\gamma}(\omega)]$, we finally obtain Eq. (C1).
* Electronic address: rxxu@ustc.edu.cn

1 H. Grabert, P. Schramm, and G. L. Ingold, Phys. Rep. 168, 115 (1988).

2 U. Weiss, Quantum Dissipative Systems, World Scientific, Singapore, 2008, 3rd ed. Series in Modern Condensed Matter Physics, Vol. 13.

3 Y. J. Yan and R. X. Xu, Annu. Rev. Phys. Chem. 56, 187 (2005).

4 A. O. Caldeira and A. J. Leggett, Physica 121A, 587 (1983).

5 G. S. Agarwal, Phys. Rev. 178, 2025 (1969), 184, 1966 (1969).

6 G. S. Agarwal, Phys. Rev. A 4, 739 (1971).

7 S. Mukamel, The Principles of Nonlinear Optical Spectroscopy, Oxford University Press, New York, 1995.

8 C. F. Klingshirn, Semiconductor Optics, Springer-Verlag, Heidelberg, 1997.

9 T. Dittrich, P. Hänggi, G. L. Ingold, B. Kramer, G. Schön, and W. Zwerger, Quantum Transport and Dissipation, Wiley-VCH, Weinheim, 1998.

10 W. H. Zurek, Rev. Mod. Phys. 75, 715 (2003).

11 P. Hänggi and G. L. Ingold, Chaos 15, 026105 (2005).

12 J. Ankerhold, H. Grabert, and P. Pechukas, Chaos 15, 026106 (2005).

13 H. Grabert, Chem. Phys. 322, 160 (2006).

14 A. Nitzan, Chemical Dynamics in Condensed Phases: Relaxation, Transfer and Reactions in Condensed Molecular Systems, Oxford University Press, New York, 2006.

15 R. P. Feynman and F. L. Vernon, Jr., Ann. Phys. (N.Y.) 24, 118 (1963).

16 Y. Tanimura, J. Phys. Soc. Jpn. 75, 082001 (2006).

17 R. X. Xu and Y. J. Yan, Phys. Rev. E 75, 031107 (2007).

18 B. L. Hu, J. P. Paz, and Y. Zhang, Phys. Rev. D 45, 2843 (1992).

19 R. Karrlein and H. Grabert, Phys. Rev. E 55, 153 (1997).

${ }^{20}$ F. Haake and R. Reibold, Phys. Rev. A 32, 2462 (1985).

21 J. J. Halliwell and T. Yu, Phys. Rev. D 53, 2012 (1996).

22 G. W. Ford and R. F. O'Connell, Phys. Rev. D 64, 105020 (2001).

23 W. G. Unruh and W. H. Zurek, Phys. Rev. D 40, 1071
(1989).

24 W. T. Strunz and T. Yu, Phys. Rev. A 69, 052115 (2004).

25 R. X. Xu, Y. Mo, P. Cui, S. H. Lin, and Y. J. Yan, in Progress in Theoretical Chemistry and Physics, Vol. 12: Advanced Topics in Theoretical Chemical Physics, edited by J. Maruani, R. Lefebvre, and E. Brändas, pages 7-40, Kluwer, Dordrecht, 2003.

26 Y. J. Yan and S. Mukamel, J. Chem. Phys. 89, 5160 (1988).

27 C. H. Chou, T. Yu, and B. L. Hu, Phys. Rev. E 77, 011112 (2008).

28 M. Grifoni and P. Hänggi, Phys. Rep. 304, 229 (1998).

29 T. Dittrich, B. Oelschlägel, and P. Hänggi, Europhys. Lett. 22, 5 (1993).

30 R. Blümel, A. Buchleitner, R. Graham, L. Sirko, U. Smilansky, and H. Walther, Phys. Rev. A 44, 4521 (1991).

31 C. Zerbe and P. Hänggi, Phys. Rev. E 52, 1533 (1995).

32 S. Kohler, T. Dittrich, and P. Hänggi, Phys. Rev. E 55, 300 (1997).

33 R. X. Xu, Y. J. Yan, Y. Ohtsuki, Y. Fujimura, and H. Rabitz, J. Chem. Phys. 120, 6600 (2004).

34 Y. Mo, R. X. Xu, P. Cui, and Y. J. Yan, J. Chem. Phys. 122, 084115 (2005).

35 A. Ishizaki and Y. Tanimura, J. Phys. Soc. Jpn. 74, 3131 (2005).

36 A. Ishizaki and Y. Tanimura, Chem. Phys. 347, 185 (2008).

37 R. X. Xu, P. Cui, X. Q. Li, Y. Mo, and Y. J. Yan, J. Chem. Phys. 122, 041103 (2005).

38 Y. A. Yan, F. Yang, Y. Liu, and J. S. Shao, Chem. Phys. Lett. 395, 216 (2004).

39 Y. Zhou and J. S. Shao, J. Chem. Phys. 128, 034106 (2008).

40 J. S. Jin, X. Zheng, and Y. J. Yan, J. Chem. Phys. 128, 234703 (2008).

41 X. Zheng, J. S. Jin, and Y. J. Yan, New J. Phys. 10, 093016 (2008).

42 J. Cao, J. Chem. Phys. 107, 3204 (1997).

43 Y. J. Yan, Phys. Rev. A 58, 2721 (1998). 